\title{
A surface-potential-based cylindrical surrounding-gate MOSFET model
}

\author{
Shuhei Amakawa, Kazuo Nakazato ${ }^{\dagger}$ and Hiroshi Mizuta ${ }^{\dagger}$ \\ Microelectronics Research Centre, Cavendish Laboratory, Madingley Road, Cambridge CB3 0HE, United Kingdom \\ Phone: +44-1223-337491, FAX: +44-1223-337706, e-mail: sa264@cam.ac.uk \\ ${ }^{\dagger}$ Hitachi Cambridge Laboratory, Cavendish Laboratory, Madingley Road, Cambridge CB3 OHE, United Kingdom
}

Conclusion: A surface-potential-based model ${ }^{1,2}$ is developed for the cylindrical surrounding-gate MOSFET (Fig. 1), of which the (non-differential) equation for the surface potential had not been known because of the cylindrical structure. Unlike other surrounding-gate MOSFET models, this model includes both drift and diffusion currents, ${ }^{3,4}$ and also there is no inherent distinction between saturation and non-saturation. ${ }^{3-5}$ Its accuracy is demonstrated by comparison with device simulation without arbitrary fitting. The model could potentially be useful as a basis for developing compact models, just as the planer single-gate counterpart ${ }^{1}$ has formed the theoretical foundations for the development of compact planar MOSFET models. ${ }^{6-8}$

Details: We formulate the model for a uniformly doped nMOS device assuming nondegeneracy using the gradual channel approximation. The gate oxide capacitance per unit length is given by $C_{\mathrm{ox}}=2 \pi \epsilon_{\mathrm{ox}} / \ln \left(1+t_{\mathrm{ox}} / a\right)$. The semiconductor charge $Q_{\mathrm{s}}$ per unit length is

$$
Q_{\mathrm{s}}(z)=-C_{\mathrm{ox}}\left[V_{\mathrm{GS}}-V_{\mathrm{fb}}-\psi_{\mathrm{s}}(z)\right],
$$

where $V_{\mathrm{GS}}$ is the gate voltage (source grounded), $V_{\mathrm{fb}}$ is the flat-band gate voltage, and $\psi_{\mathrm{s}}(z) \equiv \psi(a, z)$ is the surface potential. The semiconductor charge consists of mobile charge (electrons) and immobile charge (acceptor ions): $Q_{\mathrm{s}}=Q_{\mathrm{mo}}+Q_{\mathrm{im}}$. If we use the depletion approximation, $Q_{\mathrm{im}}=-\pi q N_{\mathrm{A}}^{-} d(2 a-d)$, where $N_{\mathrm{A}}^{-}$is the ionised acceptor density, and $d$ is the surface depletion depth. Note that the expression of $d$ for the planar MOSFET [Eq. (4) of Jang and $\mathrm{Liu}^{5}$ ] cannot be used for the cylindrical MOSFET. ${ }^{9}$ We hereafter assume that the channel is fully depleted of majority carriers (holes), leading to $Q_{\mathrm{im}} \simeq-\pi a^{2} q N_{\mathrm{A}}^{-}$.

The drain current $I_{\mathrm{D}}(z)$ is proportional to $Q_{\mathrm{mo}}(z)$ and the gradient of the electron quasi-Fermi potential $V_{\mathrm{n}}(z)$ : $I_{\mathrm{D}}(z)=-\mu_{\mathrm{n}} Q_{\mathrm{mo}}(z) \frac{\partial V_{\mathrm{n}}}{\partial z}(z)$, where $\mu_{\mathrm{n}}$ is the electron mobility, and $\frac{\partial V_{\mathrm{n}}}{\partial r}=0$ is assumed (charge-sheet approximation). This equation includes both drift and diffusion currents. In a steady state, the electron density is given by $n(r, z)=n_{\mathrm{i}} \mathrm{e}^{\beta\left[\psi(r, z)-V_{\mathrm{n}}(z)\right]}$, where $n_{\mathrm{i}}$ is the intrinsic carrier density, $\beta^{-1}=k_{\mathrm{B}} T / q$ is the thermal voltage, and $V_{\mathrm{n}}$ is the electron quasi-Fermi potential. After some algebraic manipulation, $I_{\mathrm{D}}$ can be written as

$$
\begin{aligned}
I_{\mathrm{D}}=\frac{\mu_{\mathrm{eff}} C_{\mathrm{ox}}}{L}[ & V_{\mathrm{GS}}-V_{\mathrm{fb}}-\frac{\pi a^{2} q N_{\mathrm{A}}^{-}}{C_{\mathrm{ox}}}+\frac{1}{\beta} \\
& \left.-\frac{1}{2}\left(\psi_{\mathrm{s} L}+\psi_{\mathrm{s} 0}\right)\right]\left(\psi_{\mathrm{s} L}-\psi_{\mathrm{s} 0}\right),
\end{aligned}
$$

where $\mu_{\mathrm{eff}}$ is the effective mobility, ${ }^{6}$ and $\psi_{\mathrm{s} L} \equiv \psi_{\mathrm{s}}(L)$ and $\psi_{\mathrm{s} 0} \equiv \psi_{\mathrm{s}}(0)$.

The surface potentials $\psi_{\mathrm{s} 0}$ and $\psi_{\mathrm{s} L}$ are needed to use Eq. (2). We therefore derive an equation for the surface potential. The Poisson equation for the fully depleted channel is, within the gradual channel approximation,

$$
\frac{1}{r} \frac{\partial}{\partial r}\left(r \frac{\partial \psi}{\partial r}\right)=\frac{q N_{\mathrm{A}}^{-}}{\epsilon_{\mathrm{S}}}\left[1+\mathrm{e}^{\beta\left(\psi-2 \phi_{\mathrm{F}}-V_{\mathrm{np}}\right)}\right],
$$

where $\phi_{\mathrm{F}} \simeq \beta^{-1} \ln \left(N_{\mathrm{A}}^{-} / n_{\mathrm{i}}\right)$ and $V_{\mathrm{np}}=V_{\mathrm{n}}-V_{\mathrm{p}}$ is the difference between quasi-Fermi potentials (Fig. 2). The boundary conditions for Eq. (3) are, at the centre by symmetry $\frac{\partial \psi}{\partial r}(0, z)=0$, and at the surface by Gauss' law $-2 \pi a \epsilon_{\mathrm{ox}} \frac{\partial \psi}{\partial r}(a+0, z)=Q_{\mathrm{s}}(z)$. Upon integration, we get

$r^{\prime} \frac{\partial \psi}{\partial r}\left(r^{\prime}, z\right)=\frac{q N_{\mathrm{A}}^{-}}{\epsilon_{\mathrm{S}}}\left[\frac{r^{\prime 2}}{2}+\int_{0}^{r^{\prime}} \mathrm{e}^{\beta\left(\psi-2 \phi_{\mathrm{F}}-V_{\mathrm{np}}\right)} r \mathrm{~d} r\right]$

In deriving Eq. (4), we did not make the charge-sheet assumption, and therefore the electric flux should be continuous at the interface between the channel and the gate oxide (if there is no interface trapped charge): $\epsilon_{\mathrm{Ox}} E_{r}(a+0, z)-$ $\epsilon_{\mathrm{S}} E_{r}(a-0, z)=0$, where $E_{r}(r, z)=-\frac{\partial \psi}{\partial r}(r, z)$. Then, from the second boundary condition,

$$
Q_{\mathrm{s}}(z)=2 \pi a \epsilon_{\mathrm{S}} E_{r}(a-0, z) .
$$

In order to perform the integral in Eq. (4), we use a charge-sheet model. In the charge-sheet model, mobile charge $Q_{\mathrm{mo}}(z)$ is localised to the surface, and the electric flux is discontinuous there. Note, however, that $\psi(r, z)$ is still continuous at $r=a$. The Poisson equation becomes

$$
\frac{1}{r} \frac{\partial}{\partial r}\left(r \frac{\partial \psi}{\partial r}\right)=\frac{q N_{\mathrm{A}}^{-}}{\epsilon_{\mathrm{S}}}-\frac{Q_{\mathrm{mo}}(z)}{2 \pi a \epsilon_{\mathrm{s}}} \delta(r-a) .
$$

By integrating Eq. (6) twice, we obtain

$$
\psi(r, z)=\psi_{\mathrm{s}}(z)-\frac{q N_{\mathrm{A}}^{-}}{4 \epsilon_{\mathrm{s}}}\left(a^{2}-r^{2}\right) \quad(0 \leq r<a) .
$$

The use of Eq. (7) enables us to integrate the second term of Eq. (4). By combining the resultant equation with Eqs. (1) and (5), we get

$$
\begin{aligned}
C_{\mathrm{ox}}\left[V_{\mathrm{GS}}-\right. & \left.V_{\mathrm{fb}}-\psi_{\mathrm{s}}\right]=\pi a^{2} q N_{\mathrm{A}}^{-} \\
+ & \exp \left[\beta\left(\psi_{\mathrm{s}}-\frac{a^{2} q N_{\mathrm{A}}^{-}}{4 \epsilon_{\mathrm{s}}}-2 \phi_{\mathrm{F}}-V_{\mathrm{np}}\right)\right] \\
\times & \frac{4 \pi \epsilon_{\mathrm{s}}}{\beta}\left[\exp \left(\frac{\beta a^{2} q N_{\mathrm{A}}^{-}}{4 \epsilon_{\mathrm{s}}}\right)-1\right] .
\end{aligned}
$$

Now we have the expression of the drain current [Eq. (2)] in terms of surface potentials and an equation for $\psi_{\mathrm{s}}$ [Eq. (8)]. It is, however, not entirely clear from the above derivation whether the model will be of use with reasonable accuracy.

We now compare the $I-V$ characteristics calculated from Eqs. (2) and (8) with results of device simulation. Such a comparison has to be made with care to be scientifically meaningful. We use a constant mobility both in the device simulation and in the model. We do so in order to avoid the 
(ab)use of mobility models as 'fitting functions' to obscure possible deficiencies in the basic model.

Figure 3 shows the comparison of $I_{\mathrm{D}}-V_{\mathrm{GS}}$ characteristics in log scale. The agreement is very good from subthreshold to above-threshold over ten orders of magnitude. The same result is shown in linear scale in Fig. 4 together with the relative difference between $I_{\mathrm{D}}$ from the model and from the device simulation. Figure 5 shows $I_{\mathrm{D}}-V_{\mathrm{DS}}$ characteristics with relative differences in $I_{\mathrm{D}}$. Overall, the agreement is remarkably good. Given the fact that we did not make arbitrary parameter adjustments, the observed differences in $I_{\mathrm{D}}$ are well within an expected margin. Better agreement can of course be obtained by more aggressive fitting.

${ }^{1}$ J. R. Brews, Solid-State Electron., 21, 345 (1978).

${ }^{2}$ M. Miura-Mattausch, H. J. Mattausch, N. D. Arora and C. Y. Yang, IEEE Circuits and Devices Mag., 17, 29 (2001).

${ }^{3}$ T. Endoh, T. Nakamura and F. Masuoka, IEICE Trans. Electron., E80-C, 911 (1997).

${ }^{4}$ A. Kranti, S. Haldar and R. S. Gupta, Microelectron. J., 32, 305 (2001).

${ }^{5}$ S-L. Jang and S-S. Liu, Solid-State Electron., 42, 721 (1998).

${ }^{6} \mathrm{Y}$. Tsividis, Operation and Modeling of the MOS Transistor, 2nd ed., McGraw-Hill, New York, New York, 1999.

${ }^{7}$ C. C. Enz, F. Krummenacher and E. A. Vittoz, Analog Integrated Circuits and Signal Processing, 8, 83 (1995)

8 J.-M. Sallese, M. Bucher, F. Krummenacher and P. Fazan, SolidState Electron., 47, 677 (2003).

${ }^{9}$ E. P. Pokatilov, V. M. Fomin, S. N. Balaban, V. N. Gladilin, S. N. Klimin, J. T. Devreese, W. Magnus, W. Schoenmaker, N. Collaert, M. Van Rossum and K. De Meyer, J. Appl. Phys., 85, 6625 (1999).

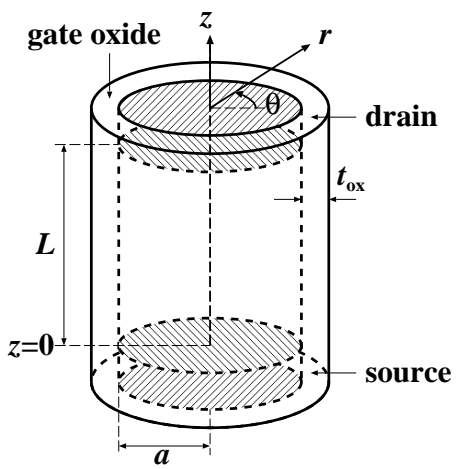

FIG. 1. A cylindrical surrounding-gate MOSFET.

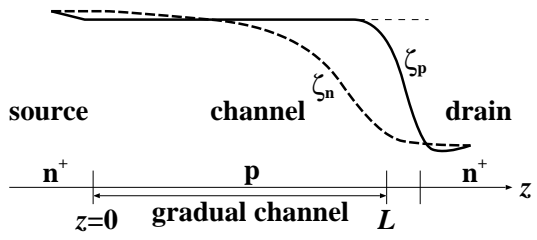

FIG. 2. Energy diagram of the customary constant quasi-Fermi level approximation for holes. $\zeta_{\mathrm{p}}=-q V_{\mathrm{p}}$ and $\zeta_{\mathrm{n}}=-q V_{\mathrm{n}}$ are the hole and electron quasi-Fermi levels, respectively. $\zeta_{\mathrm{n}}(L)-\zeta_{\mathrm{n}}(0)=-q V_{\mathrm{DS}}$ is nearly satisfied at $z=L$, where the gradual channel approximation breaks down.

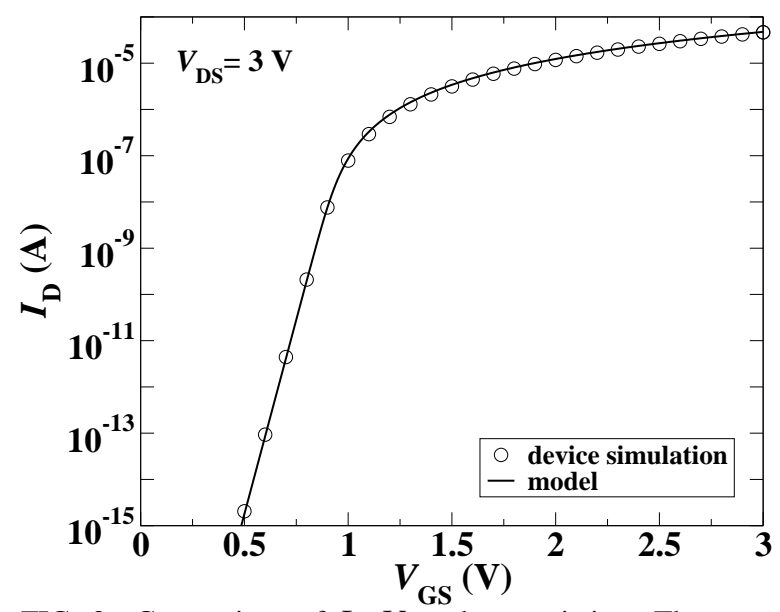

FIG. 3. Comparison of $I_{\mathrm{D}}-V_{\mathrm{GS}}$ characteristics. The parameters used are $N_{\mathrm{A}}=N_{\mathrm{A}}^{-}=1 \times 10^{16} \mathrm{~cm}^{-3}, a=50 \mathrm{~nm}$, $t_{\mathrm{ox}}=10 \mathrm{~nm}, \mu_{\mathrm{n}}=\mu_{\mathrm{eff}}=1 \times 10^{3} \mathrm{~cm}^{2} /(\mathrm{V} \cdot \mathrm{s}), T=300 \mathrm{~K}$, and $L=5 \mu \mathrm{m}$. The gate material is p-type polysilicon, and $V_{\mathrm{fb}}=0.18 \mathrm{~V}$. No arbitrary fitting is performed.

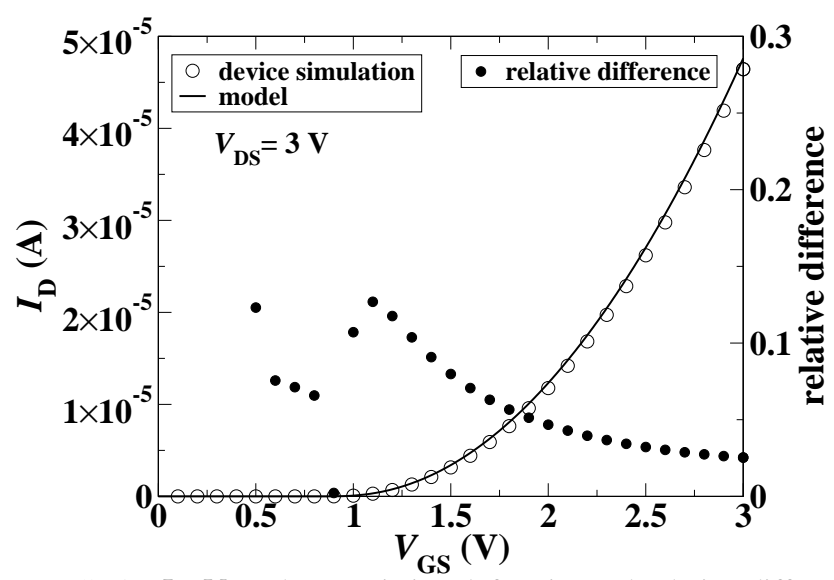

FIG. 4. $I_{\mathrm{D}}-V_{\mathrm{GS}}$ characteristics (left axis) and relative difference between $I_{\mathrm{D}}$ from the model and the device simulation (right axis).

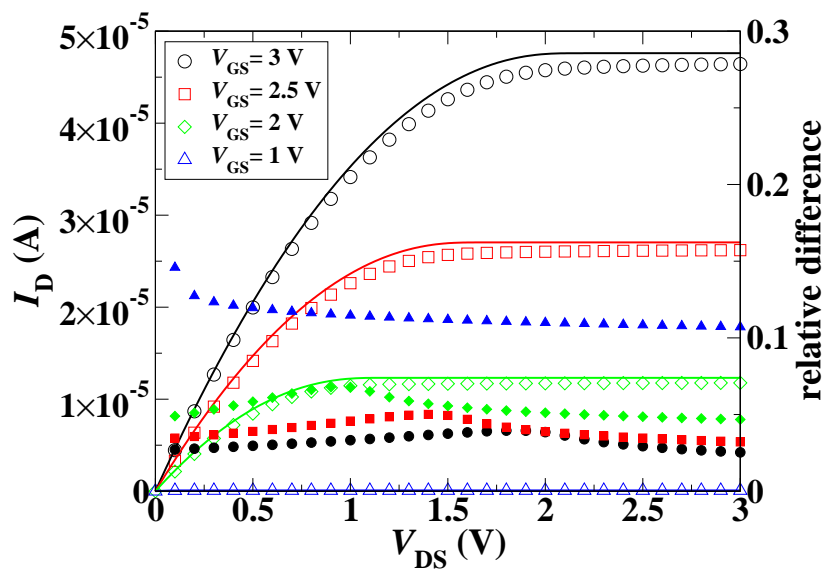

FIG. 5. $I_{\mathrm{D}}-V_{\mathrm{DS}}$ characteristics (left axis) and relative differences between $I_{\mathrm{D}}$ from the model and the device simulation (right axis). Solid lines: $I_{\mathrm{D}}$ from the model. Open symbols: $I_{\mathrm{D}}$ from the device simulation. Filled symbols: Relative differences in $I_{\mathrm{D}}$. 